\title{
The Investigation of Representation Symmetry of Free Atoms and their Atomic Energy Level Splittings in the ZnO Crystalline Field
}

\author{
Niyongabo $P$. \\ Sustainable Energy Technologies and Research (SeTAR) Centre, Faculty of engineering and the built environment, University of \\ Johannesburg, Auckland Park, South Africa
}

\begin{abstract}
Using group theoretical techniques we have investigated the representation symmetry of free dopants (Al, Ga and In) of wurtzite ZnO structure. We have also studied the symmetry of wurtzite ZnO doped with Al, Ga and In. The method allows classification of the state of dopants in the crystalline field. We show that the symmetry of free atoms is the full rotation group (FRG) and that of operators $O_{R}(\alpha, \beta, \gamma)$, where $\alpha, \beta$ and $\gamma$ are the Euler angles. The FRG is reduced to the symmetry operators of the point group of $Z n O$ $\left(C_{6 v}\right)$ when atoms are doped into the $\mathrm{ZnO}$ crystalline field. Our theoretical results confirm the available experimental data.
\end{abstract}

Keywords: Representation symmetry, irreducible representations, free atoms, crystalline field, splitting

\section{Introduction}

Symmetry has been used in many ways to assist in and illuminate the study of atomic and molecular phenomena [1]. The symmetry of matter is a powerful framework to perceive and predict the physical world. The structure of materials is described by a combination of rotations, rotation-inversions and translational symmetries. The symmetry of an object is a set of operations, called a symmetry group, which brings the object into self-congruence. The spatial symmetry operations of translation, rotation, and rotation-inversions, give rise to 32 crystallographic point groups and 230 crystallographic space groups $[2,3]$.

The lattice structure of wurtzite $\mathrm{ZnO}$ belongs to the hexagonal system with space group $\mathrm{C}_{6 \mathrm{v}}^{4}$ [4]. Based on its thermal, electrical and opto-electrical properties, $\mathrm{ZnO}$ is a material with enormous device applications. One device with promising commercial potential is a UV light-emitting diode (LED) which could be combined with phosphors to produce solid state lighting [5]. Another is a transparent field effect transistors (FET), which could serve as an active element in large area displays [6]. ZnO with wurtzite structure is naturally an n-type semiconductor because of the deviation from stoichiometry due to the presence of intrinsic defects such as $\mathrm{O}$ vacancy and $\mathrm{ZnO}$ interstitials [7]. Undoped $\mathrm{ZnO}$ shows intrinsic n-type conductivity with very high electron densities of about $10^{21} \mathrm{~cm}^{-3}[8]$.

In this paper we have investigated the representation symmetry of free atoms before and after being doped into wurtzite $\mathrm{ZnO}$ crystal. Furthermore, we study the effect of the crystalline field on the spectral terms of an atom or ion in the crystal. The studied shallow donors in wurtzite $\mathrm{ZnO}$ are $\mathrm{Al}$, $\mathrm{Ga}$ and $\mathrm{In}$, The atomic ground states of these elements are: $\mathrm{Al}$ $\left(\mathrm{n}=3,{ }^{2} \mathrm{P} \frac{1}{2}\right), \mathrm{Ga}\left(\mathrm{n}=4,{ }^{2} \mathrm{P} \frac{1}{2}\right)$ and $\mathrm{In}\left(\mathrm{n}=5,{ }^{2} \mathrm{P} \frac{1}{2}\right)$, see Fig. 5.2, p. 87 and Fig. 8.1, p. 115 in Ref. [9]. We have shown that the states of the dopants are doublets $\left(\mathrm{s}=\frac{1}{2}, 2 \mathrm{~s}+1=2\right)$ and exhibit anomalous Zeeman splitting. In addition, they become shallow donors to which excitons of symmetries $\Gamma^{\mathrm{c}}$ $\bigoplus \Gamma_{7}^{\mathrm{v}}(\mathrm{A}), \Gamma_{7}^{\mathrm{c}} \oplus \Gamma_{9}^{\mathrm{v}}(\mathrm{B})$ and $\Gamma_{7}^{\mathrm{c}} \oplus \Gamma_{7}^{\mathrm{v}}(\mathrm{C})$ are bound [10].

\section{Symmetry of a Free Atom}

The full rotation group (FRG) of a free atom follows from the solution of the Laplace's differential equation [11].

$$
\frac{\partial^{2} \varphi(x, y, z)}{\partial x^{2}}+\frac{\partial^{2} \varphi(x, y, z)}{\partial y^{2}}+\frac{\partial^{2} \varphi(x, y, z)}{\partial z^{2}}=0 \text { (1) }
$$

The resolution of Eq. (1) involves the introduction of polar coordinates in place of $\mathrm{x}, \mathrm{y}$ and $\mathrm{z}$ [12]. The transformation (rotation) which takes the points in a three-dimensional (3D) space from their initial to final positions is described in terms of Euler angles $\alpha, \beta$ and $\gamma$ and their attendant operators of FRG $\mathrm{O}_{\mathrm{R}}(\alpha, \beta, \gamma)$. These are continuous and infinite groups [11]. The basis for the operators $\mathrm{O}_{\mathrm{R}}(\alpha, \beta, \gamma)$ is the wellknown spherical harmonics $\mathrm{Y}_{\mathrm{m}}^{1}(\theta, \phi)$. The representation symmetries of free atoms are obtained from:

$\mathrm{O}_{\mathrm{R}}(\alpha, \beta, \gamma) \mathrm{Y}_{\mathrm{m}}^{1}(\theta, \phi)=\sum_{m^{\prime}} \mathrm{Y}_{\mathrm{m}}{ }^{1}(\theta, \phi) D_{\mathrm{m}^{\prime}, \mathrm{m}}^{1}(\alpha, \beta, \gamma)$

Where l-integer is the orbital angular momentum quantum number, $\mathrm{m}_{\mathrm{l}}=-1, \ldots,+\mathrm{l}$ is the orbital magnetic moment quantum number and $\mathrm{D}_{\text {m'm }}^{1}(\alpha, \beta, \gamma)$ is the matrix of $\mathrm{O}_{\mathrm{R}}(\alpha, \beta$, $\gamma)$ in the representation $D^{1}$ based on the spherical harmonics of order 1 when the spin of the electron is not taken into account. Such representation is called single value representation (SVR).

$$
\begin{aligned}
& \mathrm{D}_{\mathrm{m} \text { m }}^{1}(\alpha, \beta, \gamma)= \\
& \mathrm{e}^{-\mathrm{im} \mathrm{m}^{\prime}} \gamma \mathrm{d}_{\mathrm{m}, \mathrm{m}}^{1}(0, \beta, 0) \mathrm{e}^{-\mathrm{im}} \quad \alpha
\end{aligned}
$$

where the matrix elements of $\mathrm{D}^{1}(\alpha, \beta, \gamma)$ are:

$\mathrm{d}_{\mathrm{m}^{\prime}, \mathrm{m}}^{1}(0, \beta, 0)=$

$\sum_{t}(-1)^{t} \frac{\sqrt{\left(l+m^{\prime}\right) !\left(l-m^{\prime}\right) !(l+m) !(l-m) !}}{\left(l+m^{\prime}-t\right) !(l-m-t) !\left(t+m-m^{\prime}\right) !}$

$\times\left(\cos \frac{\beta}{2}\right)^{2 l+m^{\prime}-m-2 t} \cdot\left(\sin \frac{\beta}{2}\right)^{2 t+m-m^{\prime}}$ 


\section{International Journal of Science and Research (IJSR) \\ ISSN (Online): 2319-7064 \\ Index Copernicus Value (2013): 6.14 | Impact Factor (2015): 6.391}

and the summation is taken over all values of $t$ which lead to non-negative factorials. For example given $1=2$, for $\mathrm{d}$ configuration state of atoms with $\mathrm{m}, \mathrm{m}^{\prime}=-2,-1,0,1,2$, we obtain the reducible representation of the FRG $\mathrm{D}_{\text {m'm }}^{2}(\alpha, \beta, \gamma)=$

$\left(\begin{array}{lllll}A & B & C & D & E \\ F & G & H & I & J \\ K & L & M & N & O \\ P & Q & R & S & T \\ V & W & X & Y & Z\end{array}\right)$

where $\mathrm{A}=\mathrm{e}^{-2 i \alpha} \mathrm{d}_{22}{ }_{2} \mathrm{e}^{-2 i \gamma} \mathrm{B}=\mathrm{e}^{-2 i \alpha} \mathrm{d}_{21}^{2} \mathrm{e}^{i \gamma}$,

$\mathrm{C}=\mathrm{e}^{-2 i \alpha} \mathrm{d}_{2 \mathrm{O}}^{2}, \mathrm{D}=\mathrm{e}^{-2 i \alpha} \mathrm{d}_{2-1}^{2} \mathrm{e}^{-i \gamma}$,

$\mathrm{E}=\mathrm{e}^{-2 i \alpha} \mathrm{d}_{2-2}^{2} \mathrm{e}^{2 i \gamma} \mathrm{F}=\mathrm{e}^{-i \alpha} \mathrm{d}_{12}^{2} \mathrm{e}^{-2 i \gamma}$,

$\mathrm{G}=\mathrm{e}^{-i \alpha} \mathrm{d}_{11}^{2} \mathrm{e}^{-i \gamma} \mathrm{H}=\mathrm{e}^{-i \alpha} \mathrm{d}^{2}{ }_{10}$,

$\mathrm{I}=\mathrm{e}^{-i \alpha} \mathrm{d}_{1-1}^{2} \mathrm{e}^{i \gamma} \mathrm{J}=\mathrm{e}^{-i \alpha} \mathrm{d}_{1-2} \mathrm{e}^{2 i \gamma}$

$\mathrm{K}=\mathrm{d}_{02}^{2} \mathrm{e}^{-2 i \gamma} \mathrm{L}=\mathrm{d}_{01}^{2} \mathrm{e}^{-i \gamma}$

$\mathrm{M}=\mathrm{d}_{00 \mathrm{~N}}^{2}=\mathrm{d}_{0-1}^{2} \mathrm{e}^{i \gamma}$

$\mathrm{O}=\mathrm{d}^{2}{ }_{0-2} \mathrm{e}^{2 i \gamma} \mathrm{P}=\mathrm{e}^{i \alpha} \mathrm{d}_{-12}^{2} \mathrm{e}^{-2 i \gamma}$

$\mathrm{Q}=\mathrm{e}^{i \alpha} \mathrm{d}_{-11}^{2} \mathrm{e}^{-i \gamma} \mathrm{R}=\mathrm{e}^{i \alpha} \mathrm{d}_{-10}$

$\mathrm{S}=\mathrm{e}^{-i \alpha} \mathrm{d}_{-1-1}^{2} \mathrm{e}^{i \gamma} \mathrm{T}=\mathrm{e}^{i \alpha} \mathrm{d}_{-1-2}^{2} \mathrm{e}^{2 i \gamma}$

$(5)$

$\mathrm{V}=\mathrm{e}^{2 i \alpha} \mathrm{d}_{-22}^{2} \mathrm{e}^{-2 i \gamma} \mathrm{W}=\mathrm{e}^{2 i \alpha} \mathrm{d}_{-21}^{2} \mathrm{e}^{-i \gamma}$

$\mathrm{X}=\mathrm{e}^{2 i \alpha} \mathrm{d}^{2}{ }_{-20} \mathrm{Y}=\mathrm{e}^{2 i \alpha} \mathrm{d}_{-2-1}^{2} \mathrm{e}^{i \gamma}$

$\mathrm{Z}=\mathrm{e}^{2 i \alpha} \mathrm{d}_{-2-2}^{2} \mathrm{e}^{2 i \gamma}$

If $\alpha=\gamma=0$ (for rotation about one axis), the matrix becomes $\mathrm{D}_{\mathrm{m}, \mathrm{m}}^{2}(\beta)=$

$$
\left(\begin{array}{lllll}
d_{22}^{2} & d_{21}^{2} & d_{20}^{2} & d_{2-1}^{2} & d_{2-2}^{2} \\
d_{12}^{2} & d_{11}^{2} & d_{10}^{2} & d_{1-1}^{2} & d_{1-2}^{2} \\
d_{02}^{2} & d_{01}^{2} & d_{00}^{2} & d_{0-1}^{2} & d_{0-2}^{2} \\
d_{-12}^{2} & d_{-11}^{2} & d_{-10}^{2} & d_{-1-1}^{2} & d_{-1-2}^{2} \\
d_{-22}^{2} & d_{-21}^{2} & d_{-20}^{2} & d_{-2-1}^{2} & d_{-2-2}^{2}
\end{array}\right)
$$

where

$\mathrm{d}_{22}^{2}=\mathrm{d}_{-2-2}^{2}=\cos ^{4}\left(\frac{\beta}{2}\right)$

$\mathrm{d}_{21}^{2}=-\mathrm{d}_{12}^{2}=-\mathrm{d}_{-2-1}^{2}=\mathrm{d}_{-1-2}^{2}=\frac{1}{2} \sin [\beta(1+\cos \beta)]$

$\mathrm{d}_{20}^{2}=d_{02}^{2}=d_{-20}^{2}=\mathrm{d}_{0-2}^{2}=\sqrt{\frac{3}{8}} \sin ^{2}(\beta)$

$$
\begin{gathered}
\mathrm{d}_{2-1}^{2}=d_{1-2}^{2}=-d_{-21}^{2}=-d_{-12}^{2}=\frac{1}{2} \sin [\beta(\cos \beta-1)] \\
\mathrm{d}_{2-2}^{2}=d_{-22}^{2}=\sin ^{4}\left(\frac{\beta}{2}\right)
\end{gathered}
$$

$\mathrm{d}_{11}^{2}=d_{-1-1}^{2}=\frac{1}{2}[2 \cos (\beta)-1][\cos (\beta)+1]$

$\mathrm{d}_{1-1}^{2}=d_{-11}^{2}=\frac{1}{2}[2 \cos (\beta)+1][1-\cos (\beta)]$

$\mathrm{d}_{10}^{2}=d_{0-1}^{2}=-d_{01}^{2}=-d_{-10}^{2}=-\sqrt{\frac{3}{2}} \sin (\beta) \cos (\beta)$

$\mathrm{d}_{00}^{2}=\frac{1}{2}\left[3 \cos ^{2}(\beta)-1\right]$

If the spin $\mathrm{s}$ of an electron is taken into account for double value representations (DVR), the total angular momentum is $\mathrm{j}$-half integer $=1+\mathrm{s}$ and the matrix elements of $\mathrm{D}^{1}$ are given by

$$
\begin{aligned}
& \sum_{t}(-1) \frac{\sqrt{(j+m) !(j-m) !\left(j+m^{\prime}\right) !\left(j-m^{\prime}\right) !}}{(j+m-k) !\left(j-m^{\prime}-k\right) !\left(k+m^{\prime}-m\right) !} \\
& \times\left(\cos \frac{\beta}{2}\right)^{\mathrm{d}_{\mathrm{m}, \mathrm{m}}}(0, \beta, 0)
\end{aligned}
$$

where the summation is taken over all values of $\mathrm{k}$ which lead to non-negative factorials.

For example given $\mathrm{j}=\frac{3}{2} ; \mathrm{m}, \mathrm{m}^{\prime}=\frac{3}{2}, \frac{1}{2}, \frac{-1}{2}, \frac{-3}{2}$ we obtain

where

$$
\begin{aligned}
& \mathrm{d}_{\frac{3}{2} \frac{3}{2}}^{\frac{3}{2}}=d_{\frac{-3}{2} \frac{-3}{2}}^{\frac{3}{2}}=\cos ^{3}\left(\frac{\beta}{2}\right) \\
& \mathrm{d}_{\frac{3}{2} \frac{1}{2}}^{\frac{3}{2}}= \\
& \mathrm{d}_{\frac{-1}{2} \frac{-3}{2}}^{\frac{3}{2}}=-d_{\frac{13}{2} \frac{3}{2}}^{\frac{3}{2}}=-d_{\frac{-3}{2} \frac{-1}{2}}^{\frac{3}{2}}=-\sqrt{3} \cos ^{2}\left(\frac{\beta}{2}\right) \sin \left(\frac{\beta}{2}\right)
\end{aligned}
$$




\section{International Journal of Science and Research (IJSR) \\ ISSN (Online): 2319-7064}

Index Copernicus Value (2013): 6.14 | Impact Factor (2015): 6.391

$$
\begin{aligned}
& d_{\frac{3}{2} \frac{-1}{2}}^{\frac{3}{2}}=d_{\frac{-13}{2} \frac{3}{2}}^{\frac{3}{2}}=d_{\frac{1}{2} \frac{-3}{2}}^{\frac{3}{2}}=d_{\frac{-3}{2} \frac{1}{2}}^{\frac{3}{2}}=\sqrt{3} \cos \left(\frac{\beta}{2}\right) \sin \left(\frac{\beta}{2}\right) \\
& \mathrm{d}_{\frac{3}{2} \frac{-3}{2}}^{\frac{3}{2}}=\mathrm{d}_{\frac{-3}{2} \frac{3}{2}}^{\frac{3}{2}}=-\sin ^{3}\left(\frac{\beta}{2}\right)(10) \\
& \mathrm{d}_{\frac{1}{2} \frac{1}{2}}^{\frac{3}{2}}=\mathrm{d}_{\frac{-1-1}{2} \frac{1}{2}}^{\frac{3}{2}}=\cos \left(\frac{\beta}{2}\right)\left[3 \cos ^{2}\left(\frac{\beta}{2}-2\right)\right] \\
& \mathrm{d}_{\frac{1}{2} \frac{-1}{2}}^{\frac{3}{2}}=-\mathrm{d}_{\frac{-13}{2} \frac{3}{2}}^{\frac{3}{2}}=\sin \left(\frac{\beta}{2}\right)\left[3 \sin ^{2}\left(\frac{\beta}{2}-2\right)\right]
\end{aligned}
$$

Since the character of a rotation depends only on the angle of rotation and not on the direction of the rotation axis, we can find the characters of $\mathrm{D}^{1}$ and $\mathrm{D}^{\mathrm{j}}$ using

$$
\chi^{l}(\beta)=\frac{\sin \left[\left(l+\frac{1}{2}\right) \beta\right]}{\sin \left(\frac{\beta}{2}\right)}
$$

for SVR and

$$
\chi^{j}(\beta)=\frac{\sin \left[\left(j+\frac{1}{2}\right) \beta\right]}{\sin \left(\frac{\beta}{2}\right)}
$$

for DVR. Where the angle $\beta$ is an arbitrary angle of rotation about any direction of the rotation axis. We have considered the symmetry of free atoms and the classification of their states according to reducible $\mathrm{D}^{1}$ and $\mathrm{D}^{\mathrm{j}}$ of the FRG.

\section{Splitting of atomic energy levels of $\mathrm{Al}$, Ga and In, in the crystalline field of $\mathrm{ZnO}$ point group}

In order to highlight that the $\mathrm{ZnO}$ is naturally doped by $\mathrm{Al}$, $\mathrm{Ga}$ and In, we have shown and tabulated six excitonic transition lines $\mathrm{I}_{6 \mathrm{a}}, \mathrm{I}_{8}, \mathrm{I}_{9}$ and $\mathrm{I}_{0}, \mathrm{I}_{1}, \mathrm{I}_{2}$, where $\mathrm{I}_{6 \mathrm{a}}, \mathrm{I}_{8}$ and $\mathrm{I}_{9}$ are neutral exciton complexes bound to $\mathrm{Al}, \mathrm{Ga}$ and In impurity of $\mathrm{ZnO}$, respectively [10]. These lines are accompanied by higher energetic lines $I_{2}, I_{1}$, and $I_{0}$. The neutral bound excitonic line $\mathrm{I}_{6 \mathrm{a}}$ was correlated to $\mathrm{I}_{1}, \mathrm{I}_{8}$ was correlated to $\mathrm{I}_{1}$ and $I_{9}$ was correlated to $I_{2}$ (see Fig. 1 in Ref. [10]).

When an atom is doped into a crystal, its electron will be perturbed by the crystalline field, i.e., since an electric field is produced at the position of the atom by all other atoms in the crystal, according to many authors $[13,14]$. The electric field has the symmetry of one of the crystal point groups. In this case, the energy levels of unperturbed system will be classified according to the representations of the FRG. The level belonging to 1-representation will be $(21+1)$-fold degeneracy for SVR and $(2 \mathrm{j}+1)$-fold degeneracy for DVR [15]. For wurtzite $\mathrm{ZnO}$, the crystal point group is $\mathrm{C}_{6 \mathrm{v}}$ [16]. The characters for SVR and DVR are calculated and tabulated in tables 1 and 3 respectively for $C_{6 v}$. Using the Eqs, (11) and (12) we obtain the characters of the rotation groups of the free atoms in $\mathrm{D}^{1}$ and $\mathrm{D}^{\mathrm{j}}$ representations (see tables 2 and 4).
Table 1: SVR character table for $\mathrm{C}_{6 \mathrm{v}}$ using CDML labelling

\begin{tabular}{|c|c|c|c|c|c|c|}
\hline $\mathbf{C}_{\mathbf{6 v}}$ & $\mathbf{E}$ & $\begin{array}{c}\mathbf{C}_{\mathbf{6}} \\
\mathbf{( 3 )}\end{array}$ & $\begin{array}{c}\mathbf{C}_{\mathbf{6}}^{\mathbf{2}} \\
\mathbf{( 2 )}\end{array}$ & $\begin{array}{c}\mathbf{C}_{\mathbf{6}} \\
\mathbf{( 2 )}\end{array}$ & $\begin{array}{c}\sigma_{\mathbf{v}} \\
\mathbf{( 3 )}\end{array}$ & $\begin{array}{c}\sigma_{\mathbf{v}} \\
\mathbf{( 3 )}\end{array}$ \\
\hline$\Gamma_{1}$ & 1 & 1 & 1 & 1 & 1 & 1 \\
\hline$\Gamma_{2}$ & 1 & 1 & 1 & 1 & -1 & -1 \\
\hline$\Gamma_{3}$ & 1 & -1 & 1 & -1 & 1 & -1 \\
\hline$\Gamma_{4}$ & 1 & -1 & 1 & -1 & -1 & 1 \\
\hline$\Gamma_{5}$ & 2 & 2 & -1 & -1 & 0 & 0 \\
\hline$\Gamma_{6}$ & 2 & -2 & -1 & 1 & 0 & 0 \\
\hline
\end{tabular}

\section{Discussions}

For the orbital angular momentum 1, the spectral terms of an atom are classified according to $\operatorname{SVR}\left(D_{m}^{1}{ }_{m}^{\prime}(\alpha, \beta, \gamma)\right)$ of the FRG of the atom; while for the total angular momentum $\mathrm{j}$, the states are classified according to $\operatorname{DVR}\left(\mathrm{D}^{\mathrm{j}}{ }_{\mathrm{m}} \mathrm{m}(\alpha, \beta, \gamma)\right)$. The $D^{1}$ and $D^{j}$ representations are reduced into irreducible representations of $\mathrm{C}_{6 \mathrm{v}}$ by means of the reduction formulas:

$$
\begin{aligned}
& \mathrm{a}_{\mu}=\frac{1}{g} \sum_{\mu} g_{i} \chi_{i}^{l}(\beta) \chi_{i}^{(\mu)}(\beta) \\
& \mathrm{a}_{\sigma}=\frac{1}{g} \sum_{\sigma} g_{i} \chi_{i}^{j}(\beta) \chi_{i}^{(\sigma)}(\beta)
\end{aligned}
$$

For l-integer and $\mathrm{j}$-half integer, respectively. The $\mathrm{g}$ and $\mathrm{g}_{\mathrm{i}}$ stand for the order of the point group and the number of the symmetry operators in a class, respectively. The $\chi^{1}$ and $\chi^{\mathrm{j}}$ stand for the characters of the FRG $\mathrm{O}_{\mathrm{R}}(\alpha, \beta, \gamma)$; and $\chi \mu$ and $\chi \sigma$ are characters of a point group of the crystalline field. $\mu \equiv \Gamma_{1}, \Gamma_{2} \ldots \Gamma_{6}$ and $\sigma \equiv \Gamma_{7}, \Gamma_{8}, \Gamma_{9}$.

The characters $\chi^{1}(\beta)$ and $\chi^{\mathrm{j}}(\beta)$ are listed in the tables 2 and 4 , respectively, with the characters of $\Gamma_{\mathrm{i}}(\mathrm{i}=1,2,3, \ldots$, 6) and $\Gamma_{\mathrm{k}}(\mathrm{k}=7,8,9)$ tabulated in tables 1 and 3 , respectively.

Using the reduction formulas in Eqs. (13) and (14), we determined the Zeeman splittings of the spectral terms of the free atoms and for greater understanding we have shown that if the free atoms are doped into $\mathrm{ZnO}$ crystal, then levels split into new terms belonging to the irreducible representations of the crystal point group $\mathrm{C}_{6 \mathrm{v}}$ (see tables 2 and 4 last columns).

Table 2: Characters of classes of $\mathrm{C}_{6 \mathrm{v}}$ in the $(21+1)$ dimensional representation $\mathrm{D}^{1}$ and the resolution of $\mathrm{D}^{1}$ into irreducible representation of $\mathrm{C}_{6 \mathrm{v}}$ using CDML labelling [4].

\begin{tabular}{|c|c|c|c|c|c|c|c|}
\hline $\mathbf{I}$ & $\mathbf{E}$ & $\mathbf{C 6}^{\mathbf{3}}$ & $\begin{array}{c}\mathbf{C 6} \\
\mathbf{( 2 )}\end{array}$ & $\begin{array}{c}\mathbf{C 6} \\
\mathbf{( 2 )}\end{array}$ & $\begin{array}{c}\sigma_{v} \\
\mathbf{( 3 )}\end{array}$ & $\begin{array}{c}\sigma_{v} \\
\mathbf{( 3 )}\end{array}$ & \\
\hline 0 & 1 & 1 & 1 & 1 & 1 & 1 & Splittings \\
\hline 1 & 3 & -1 & 0 & -1 & -1 & -1 & $\mathrm{D}^{0}=\Gamma_{1}$ \\
\hline 2 & 5 & 1 & -1 & 1 & 1 & 1 & ${ }^{1}=\Gamma_{2}+\Gamma_{5}$ \\
\hline 3 & 7 & -1 & 1 & -1 & -1 & -1 & $\mathrm{D}^{2}=\Gamma_{1}+\Gamma_{5}+\Gamma_{6}$ \\
\hline 4 & 9 & 1 & 0 & -2 & 1 & 1 & ${ }^{3}=\Gamma_{2}+\Gamma_{3}+\Gamma_{4}+\Gamma_{5}+\Gamma_{6}$ \\
\hline 5 & 11 & -1 & -1 & -1 & -1 & -1 & ${ }^{5}+\Gamma_{3}+\Gamma_{4}+\Gamma_{5}+2 \Gamma_{6}$ \\
\hline 6 & 13 & 1 & 1 & 1 & 1 & 1 & ${ }^{6}=2 \Gamma_{2}+\Gamma_{3}+\Gamma_{4}+2 \Gamma_{5}+2 \Gamma_{2}+\Gamma_{3}+\Gamma_{4}+2 \Gamma_{5}+2 \Gamma_{6}$ \\
\hline
\end{tabular}




\section{International Journal of Science and Research (IJSR) \\ ISSN (Online): 2319-7064}

Index Copernicus Value (2013): 6.14 | Impact Factor (2015): 6.391

Table 3: DVR character table for the double point group $\mathrm{C}_{6 \mathrm{v}}$ using CDML labelling [4]

\begin{tabular}{|l|l|l|l|l|l|l|l|l|l|}
\hline & & & $\mathbf{C}_{\mathbf{6}} \mathbf{C}_{6}{ }^{4}$ & $\mathbf{C}_{6}$ & $\mathbf{C}_{\mathbf{6}}{ }^{2}$ & $\mathbf{C}_{\mathbf{6}}{ }^{5}$ & $\sigma_{v}(\mathbf{3})$ & $\sigma_{v^{\prime}}(\mathbf{3})$ \\
\hline $\mathrm{C}_{6 \mathrm{v}}$ & $\mathrm{E}$ & $\mathrm{R}$ & $\begin{array}{l}\mathrm{C}_{6}{ }^{3} \\
\mathrm{R}\end{array}$ & $\begin{array}{l}\mathrm{C}_{6}{ }^{2} \\
\mathrm{R}\end{array}$ & $\begin{array}{l}\mathrm{C}_{6}{ }^{5} \\
\mathrm{R}\end{array}$ & $\begin{array}{l}\mathrm{C}_{6}{ }^{4} \\
\mathrm{R}\end{array}$ & $\begin{array}{l}\mathrm{C}_{6} \\
\mathrm{R}\end{array}$ & $\begin{array}{l}\sigma_{v} \\
\mathrm{R}(3)\end{array}$ & $\begin{array}{l}\sigma_{v^{\prime}}{ }^{\prime} \\
\mathrm{R}(3)\end{array}$ \\
\hline$\Gamma_{7}$ & 2 & -2 & 0 & 1 & $\sqrt{3}$ & -1 & $-\sqrt{3}$ & 0 & 0 \\
\hline$\Gamma_{8}$ & 2 & -2 & 0 & 1 & $-\sqrt{3}$ & -1 & $\sqrt{3}$ & 0 & 0 \\
\hline$\Gamma_{9}$ & 2 & -2 & 0 & -2 & 0 & 2 & 0 & 0 & 0 \\
\hline
\end{tabular}

Consider for example $1=2$ and $\mu \equiv \Gamma_{1}, \Gamma_{5}$ and $\Gamma_{6}$.

For $\mu \equiv \Gamma_{1}$ :

$$
\frac{1}{12}[(5 \times 1)+(1 \times 1)+2(-1 \times 1)+2(1 \times 1)+3(1 \times 1)+
$$

$3(1 \times 1)]=1$

For $\mu \equiv \Gamma_{5}$ :

$\frac{1}{12}[(5 \times 2)+(1 \times 2)+2(-1 \times-1)+2(1 \times-1)+3(1 \times 0)+$

$3(1 \times 0)]=1$

For $\mu \equiv \Gamma_{6}$

$\frac{1}{12}[(5 \times 2)+(1 \times-2)+2(-1 \times-1)+2(1 \times 1)+3(1 \times 0)+$ $3(1 \times 1)]=1$

We conclude that $\mathrm{D}^{2}=\Gamma_{1+} \Gamma_{5}+\Gamma_{6}$. The d-state of free atoms splits into $\Gamma_{1}, \Gamma_{5}$ and $\Gamma_{6}$ belonging to SVR of $\mathrm{C}_{6 \mathrm{v}}$ (see the last column, fourth row of Table 2).

Table 4: Characters of $\mathrm{C}_{6 \mathrm{v}}$ in the $(2 \mathrm{j}+1)$-dimensional representation and the resolution of $\mathrm{D}^{\mathrm{j}}$ into irreducible representations of $\mathrm{C}_{6 \mathrm{v}}$ using CDML labelling [4], where $\mathrm{K}_{\mathrm{i} \text {; }}$

$1,2,3, \ldots, 9$ stands for symmetry operators of the double point group $\mathrm{C}_{6 \mathrm{v}}$.

\begin{tabular}{|c|c|c|c|c|c|}
\hline $\mathrm{j}$ & $\mathrm{K}_{1}$ & $\mathrm{~K}_{2}$ & $\mathbf{K}_{\mathbf{3}}$ & $\mathbf{K}_{4}$ & Splittings \\
\hline 1 & \multirow[t]{2}{*}{2} & \multirow[t]{2}{*}{-2} & \multirow[t]{2}{*}{1} & \multirow[t]{2}{*}{$\mathbf{0}$} & 1 \\
\hline 2 & & & & & D $=I_{7}$ \\
\hline 3 & \multirow[t]{2}{*}{4} & \multirow[t]{2}{*}{-4} & \multirow[t]{2}{*}{$\mathbf{0}$} & \multirow[t]{2}{*}{-1} & 3 \\
\hline 2 & & & & & $\mathbf{D}^{2}=\Gamma_{7}+\Gamma_{9}$ \\
\hline 5 & \multirow[t]{2}{*}{6} & \multirow[t]{2}{*}{-6} & \multirow[t]{2}{*}{$\mathbf{0}$} & \multirow[t]{2}{*}{$\mathbf{0}$} & $\frac{5}{-}>>$ \\
\hline 2 & & & & & $D^{\overline{2}}=\Gamma_{7}+\Gamma_{8}+\Gamma_{9}$ \\
\hline 7 & \multirow[t]{2}{*}{8} & \multirow[t]{2}{*}{-8} & \multirow[t]{2}{*}{$\mathbf{0}$} & \multirow[t]{2}{*}{1} & $\underline{7}$ \\
\hline$\overline{2}$ & & & & & $\mathbf{D}^{\overline{2}}=\Gamma_{7}+2 \Gamma_{8}+\Gamma_{9}$ \\
\hline$\underline{9}$ & \multirow[t]{2}{*}{10} & \multirow[b]{2}{*}{10} & \multirow[t]{2}{*}{$\mathbf{0}$} & \multirow[t]{2}{*}{-1} & $\underline{9}$ \\
\hline$\overline{2}$ & & & & & $\mathbf{D}^{2}=\Gamma_{7}+2 \Gamma_{8}+2 \Gamma_{9}$ \\
\hline 11 & \multirow[t]{2}{*}{12} & \multirow{2}{*}{$\overline{12}$} & \multirow[t]{2}{*}{$\mathbf{0}$} & \multirow[t]{2}{*}{$\mathbf{0}$} & $\underline{11}$ \\
\hline 2 & & & & & $\mathrm{D}^{\overline{2}}=2 \Gamma_{7}+2 \Gamma_{8}+2 \Gamma_{9}$ \\
\hline
\end{tabular}

Continued. Characters of $\mathrm{C}_{6 \mathrm{v}}$ in the $(2 \mathrm{j}+1)$-dimensional representation and the resolution of $\mathrm{D}^{\mathrm{j}}$ into irreducible representations of $\mathrm{C}_{6 \mathrm{v}}$ using CDML labelling [4], where $\mathrm{K}_{\mathrm{i} ; \mathrm{i}}=$ $1,2,3 \ldots, 9$ stands for symmetry operators of the double point group $\mathrm{C}_{6 \mathrm{v}}$.

\begin{tabular}{|c|c|c|c|c|c|c|}
\hline $\mathbf{j}$ & $\mathbf{K}_{\mathbf{5}}$ & $\mathbf{K}_{\mathbf{6}}$ & $\mathbf{K}_{\mathbf{7}}$ & $\mathbf{K}_{\mathbf{8}}$ & $\mathbf{K}_{9}$ & Splittings \\
\hline$\frac{1}{2}$ & $\sqrt{3}$ & $\mathbf{- 1}$ & $-\sqrt{3}$ & $\mathbf{0}$ & $\mathbf{0}$ & $\mathbf{D}^{\frac{1}{2}}=\boldsymbol{\Gamma}_{\mathbf{7}}$ \\
\hline$\frac{3}{2}$ & $\sqrt{3}$ & $\mathbf{1}$ & $-\sqrt{3}$ & $\mathbf{0}$ & $\mathbf{0}$ & $\mathbf{D}^{\frac{3}{2}}=\boldsymbol{\Gamma}_{7}+\boldsymbol{\Gamma}_{\mathbf{9}}$ \\
\hline$\frac{5}{2}$ & $\mathbf{0}$ & $\mathbf{0}$ & $\mathbf{0}$ & $\mathbf{0}$ & $\mathbf{0}$ & $\mathbf{D}^{\frac{5}{2}}=\boldsymbol{\Gamma}_{\mathbf{7}}+\boldsymbol{\Gamma}_{\mathbf{8}}+\boldsymbol{\Gamma}_{\mathbf{9}}$ \\
\hline$\frac{7}{2}$ & $-\sqrt{3}$ & $\mathbf{1}$ & $\sqrt{3}$ & $\mathbf{0}$ & $\mathbf{0}$ & $\mathbf{D}^{\frac{7}{2}}=\boldsymbol{\Gamma}_{\mathbf{7}}+\mathbf{2} \boldsymbol{\Gamma}_{\mathbf{8}}+\boldsymbol{\Gamma}_{\mathbf{9}}$ \\
\hline$\frac{9}{2}$ & $-\sqrt{3}$ & $\mathbf{1}$ & $\sqrt{3}$ & $\mathbf{0}$ & $\mathbf{0}$ & $\frac{9}{\frac{9}{2}}=\boldsymbol{\Gamma}_{\mathbf{7}}+\mathbf{2} \boldsymbol{\Gamma}_{\mathbf{8}}+\mathbf{2} \boldsymbol{\Gamma}_{\mathbf{9}}$ \\
\hline$\frac{11}{2}$ & $\mathbf{0}$ & $\mathbf{0}$ & $\mathbf{0}$ & $\mathbf{0}$ & $\mathbf{0}$ & $\mathbf{D}^{\frac{11}{2}}=\mathbf{2} \boldsymbol{\Gamma}_{\mathbf{7}}+\mathbf{2} \boldsymbol{\Gamma}_{\mathbf{8}}+\mathbf{2} \boldsymbol{\Gamma}_{\mathbf{9}}$ \\
\hline
\end{tabular}

Consider $\mathrm{j}=\frac{3}{2}$ and $\sigma \equiv \Gamma_{7}$ and $\Gamma_{9}$

For $\sigma \equiv \Gamma_{7}$

$\frac{1}{24}[(4 \times 2)+(-4 \times-2)+2(0 \times 0)+2(-1 \times 1)+$

$2(\sqrt{3} \times \sqrt{3})+2(1 \times-1)+2(-\sqrt{3} \times-\sqrt{3})+6(0 \times 0)+$

$6(0 \times 0)]=1$

For $\sigma \equiv \Gamma_{9}$

$\frac{1}{24}[(4 \times 2)+(-4 \times-2)+2(0 \times 0)+2(-1 \times-2)+2(\sqrt{3} \times 0)+$

$2(1 \times 2)+2(-\sqrt{3} \times 0)+6(0 \times 0)+6(0 \times 0)]=1$

The $\mathrm{j}=\frac{3}{2}$ level splits into $\Gamma_{7}$ and $\Gamma_{9}$ symmetries of $\mathrm{C}_{6 \mathrm{v}}$.

$\mathrm{D} \frac{3}{2}=\Gamma_{7}+\Gamma_{9}$ (see the last column, third row of Table 4$)$.

More results regarding the splitting of atomic levels when atoms are doped into $\mathrm{ZnO}$ crystal are tabulated in Table 2 and 4.

\section{Conclusion}

$\mathrm{ZnO}$ is naturally n-type semiconductor. The shallow donors $\mathrm{Al}, \mathrm{Ga}$ and In have been studied. The symmetry of the free atoms is FRG. When these atoms are substitutionally placed to $\mathrm{Zn}$ sites of the $\mathrm{ZnO}$ crystal point group $\mathrm{C}_{6 \mathrm{v}}$. Using the characters of $\mathrm{C}_{6 \mathrm{v}}$ and of $\mathrm{D}^{1}$ and $\mathrm{D}^{\mathrm{j}}$ in 1 and $\mathrm{j}$ representations, respectively, by means of the reduction formulas in Eqs. (13) and (14), we find the number of times that each irreducible representation of $\mathrm{C}_{6 \mathrm{v}}$ is contained in the 1 and $\mathrm{j}$ representations. Thus we determine how the degenerate level splits in the crystalline field.

\section{Acknowledgements}

It is a pleasure to acknowledge the very fruitful discussions with Dr Fred J. Nambala for his contributions and support in the preparation of the manuscript. 


\section{References}

[1] Berry R. S. SYMMETRY IN ATOMIC AND MOLECULAR SYSTEMS. (1991). Computers Math. Applic. 21, pp. 39-52.

[2] Burns G. and Glazer A.M. (1990). Space Groups for Solid State Scienticists. Academic.

[3] Opechowski W. (1986). Crystallographic and Meta crystallographic Groups. North-Holland.

[4] Cracknell A.P., Davies B.L., Miller S.C., Love W.F. (CDML). (1979). Kronecker Product Tables 1-4 .ifl/Plenum, New York, London.

[5] Zhang Y.et.al. (2012). Synthesis, Characterization, and Applications of $\mathrm{ZnO}$ Nanowires. Journal of Nanomaterials. 2012, pp. 22.

[6] Ozgur U. et al. (2005). A comprehensive review of $\mathrm{ZnO}$ materials and devices. Journal of Applied Physics. 98, pp. 041301.

[7] Walukiewicz W. (1994). Defect formation and diffusion in heavily doped semiconductors. Phys.Rev. B 50, pp. 5221.

[8] Minami T., Sato H., Nanto H. and Takata S. (1995). Group III impurity doped $\mathrm{Zn}$ Oxide thin films prepared by RF magnetron sputtering. J.Applied.Phys. 24, pp. 3192.

[9] White H.E. (1934). Introduction to Atomic Spectra. McCraw-Hill, New York.

[10] Wagner M.R., Kunert H.W., Machatine A.G.J., Niyongabo P., Malherbe J.B., Hoffmann A., Barnas J. (2009). Bound and free excitons in $\mathrm{ZnO}$. Optical selection rules in the absence and presence of time reversal symmetry. Microelectronics Journal. 40, pp. 289-292.

[11] Hamermesh M. (1962). Group theory and its application to physical problems. Addison-Wesley.

[12] Wigner E.P. (1959). Group Theory and its Application to Quantum Mechanics of Atomic Spectra. Academic Press, New York, London.

[13] Yu P.Y. and Cardona M. (2010). Fundamentals of Semiconductors. Springer.

[14] Sugana S., Tanabe Y. and Kamimura H. (1970). Multiplets of Transition-Metal Ions in Crystals. Academic Press, New York, London.

[15] Tinkham M. (1964). Group Theory and Quantum Mechanics. Megraw-Hill Book Company New York, San Francisco, Toronto, London.

[16] Koster G.F., Dimmock J.O., Wheeler R.C. and Statz H. (1963). Properties of the Thirty-Two Point Groups. MIT Press, Cambridge. 